Paula Francezca C. Padua, MD ${ }^{1}$

Arik Paolo Isaiah C. Dela Cruz, M.D., ${ }^{1,2}$

Renato C. Pascual, Jr, MD'

Steve Marlo M. Cambe, MD'

${ }^{1}$ Department of Otolaryngology

Head and Neck Surgery

St. Luke's Medical Center, Q.C.

279 E. Rodriguez Sr. Blvd. Quezon City, Philippines

${ }^{2}$ Department of Otolaryngology

Head and Neck Surgery

St. Gabriel Hospital

Dr. Rafael S. Tumbukon Memorial Hospital

Aklan, Philippines

\section{Usability of a Smartphone Application for Pre-operative Facial Analysis for Rhinoplasty among ENT Surgeons}

\author{
ABSTRACT \\ Objective: To determine the usability of a smartphone application (ImageMeter) by ENT \\ surgeons for pre-operative photographic analysis of facial angles for rhinoplasty using the USE \\ questionnaire.

\section{Methods:}

$\begin{array}{ll}\text { Design: } & \text { Post-Test Only Non-Experimental Evaluation Study } \\ \text { Setting: } & \text { Tertiary Private Training Hospital Outpatient Clinic } \\ \text { Participants: } & \text { Twenty-five (25) ENT residents and consultants }\end{array}$

Results: Of 45 ENT surgeons invited, 25 ENT residents and consultants (16 males, 9 females) aged 28 to 52 years old (mean age 36 years old) trialed the use of the Image Meter application in measuring the naso-facial, naso-frontal and naso-labial angles of pre-selected lateral images of 10 volunteers and completed our survey. The usability of the application was measured using the USE questionnaire, through usefulness (Cronbach $\alpha=0.99$ ), ease of use ( $\alpha=0.85$ ), ease of learning $(\alpha=0.66)$ and satisfaction $(\alpha=0.69)$. On a scale of 1-7, results showed that for the ENT surgeons surveyed, the app was generally useful $(M=6.10, S D=0.73)$, easy to use $(M=6.13, S D=0.63)$, easy to learn $(M=6.31, S D=0.62)$ and satisfactory $(M=6.06, S D=0.7)$. As for overall outcome, the ENT surgeons found the application usable $(M=6.15, S D=0.11)$.

Conclusion: When applied to human facial analysis, the ImageMeter measurement of angles feature may be a usable tool for ENT surgeons in the pre-operative evaluation of patients undergoing rhinoplasty. Based on USE questionnaire responses, it is easy to use, quick to learn, useful and satisfactory in the preoperative measurement of facial angles.

Keywords: imageMeter; photography; esthetic; rhinoplasty; face; anthropometry

The human nose is the most prominent and central feature of the face, and rhinoplasty is thus considered one of the most challenging facial plastic surgical procedures, requiring meticulous pre-operative analysis and understanding of the patient's needs and expectations. ${ }^{1-3}$ Preoperative planning, has evolved over the years and includes manual anthropometry wherein 
ORIGINAL ARTICLES

surface measurements are taken with calipers and flexible measuring tape, and 2D photography which entails taking photos of patients with their head positioned in special orientation with their Frankfort plane horizontal to the ground. ${ }^{4}$ Recently, digital photography has made photographic documentation easier more effective and economical when compared to conventional photography. ${ }^{5}$ Free, downloadable smartphone applications have made an impact on the practice of medicine and have become available to measure angles in these photos. ${ }^{6-10}$

The ImageMeter is a free, downloadable smartphone application, originally designed for home/office construction and planning that lets you measure dimensions, angles and areas in your photos with ease. ${ }^{11}$ However, to the best of our knowledge based on an extensive search of PubMed (MEDLINE), EMBASE, HERDIN and Google Scholar using the search terms "rhinoplasty," "facial angles" "photography AND facial esthetics," "ease of use," it has not been used to measure facial angles for rhinoplasty evaluation.

This study aimed to determine the usability of the ImageMeter smartphone application by ENT surgeons for pre-operative photographic analysis of facial angles for rhinoplasty using the USE questionnaire.

\section{METHODS}

With institutional review committee approval, this Post-Test Only Non-Experimental Evaluation Study was conducted among ENT surgeons and trainees at the outpatient clinic of the St. Luke's Medical Center Quezon City from November 6 - 10, 2017.

After obtaining informed consent, respondents trialed use of the Image Meter on pre-determined lateral photographs of patient volunteers and subsequently responded to questionnaires on the usability of a smartphone-based application for measurement of facial angles.

The ImageMeter version 2.19.1 (Dirk Farin Kronenstr.49b 70174 Stuttgart, Germany) application for facial angle analysis was used in this survey. Standard photographic lateral views of ten (10) patient volunteers stored in the researcher's android smartphone (Samsung Galaxy S7 Edge, SM-G935F, Samsung Electronics, New Jersey, USA) were retrieved. (Figure 1) Standard views had been taken at the volunteer's eye level at a distance of 2 feet with the volunteer being exactly $90^{\circ}$ from the lens using the Frankfort horizontal line as a guide (except for the basal view) against a solid blue background. Of the standardized views for photographing patients undergoing rhinoplasty (anteroposterior, right and left lateral, right and left oblique and basal), only the left lateral views were utilized for purposes of this study with written informed consent for use of their photos in full for the study and for subsequent publication provided by all ten volunteers. For each lateral image, the following facial landmarks were identified (Figure 2):

Nasofacial angle: nasion, pronasale, pogonion

Nasofrontal angle: glabella, pronasale, nasion

Nasolabial angle: columella, upper lip

General introduction to the application and demonstration on its use was conducted at the outpatient clinic by one researcher using a single narrative. Each surgeon or trainee participated individually. The respondents were informed of the basic services offered by the application, the tasks that may be performed and how users can benefit from the application in general. Using the application's angle option, each participant placed a point on and connected the facial landmarks per facial angle measured. The landmarks were adjusted as the respondent pleased, and the angle generated automatically adjusted accordingly. A single researcher ensured that each respondent could use the app correctly allowing several attempts before completing the trial on the 10 stored photos. After completing the trial on the 10 stored photos, each respondent was asked to answer the USE questionnaire with no time limit to complete the survey. Completion of the introduction, demonstration, trial of the app and answering the questionnaire was performed individually upon the availability of the researcher and participant. Pre-testing of the questionnaire was not performed.

The USE Questionnaire (Figure 3) is a free, standardized tool to determine the usability of software, hardware, services and user support materials using 4 domains - - ease of use, ease of learning, and satisfaction -- as dependent variables. ${ }^{12}$ It contains 30 questions organized under the headings ease of use (8 items), satisfaction (11 items), usefulness ( 4 items) and ease of learning ( 7 items) each item followed by a seven-point Likert scale ranging from "strongly disagree" to "strongly agree," plus a "not applicable" (NA) option.

\section{Data Analysis}

Data on respondents and their responses was compiled and analyzed using Microsoft ${ }^{\oplus}$ Excel for Mac v. 15.22 (160506) (Microsoft Corp., Redmond, WA, USA). Descriptive statistics (mean and standard deviation) were computed for respondent data. Internal consistency was computed for the USE Questionnaire responses using Cronbach's $\alpha$. 


\section{ORIGINAL ARTICLES}

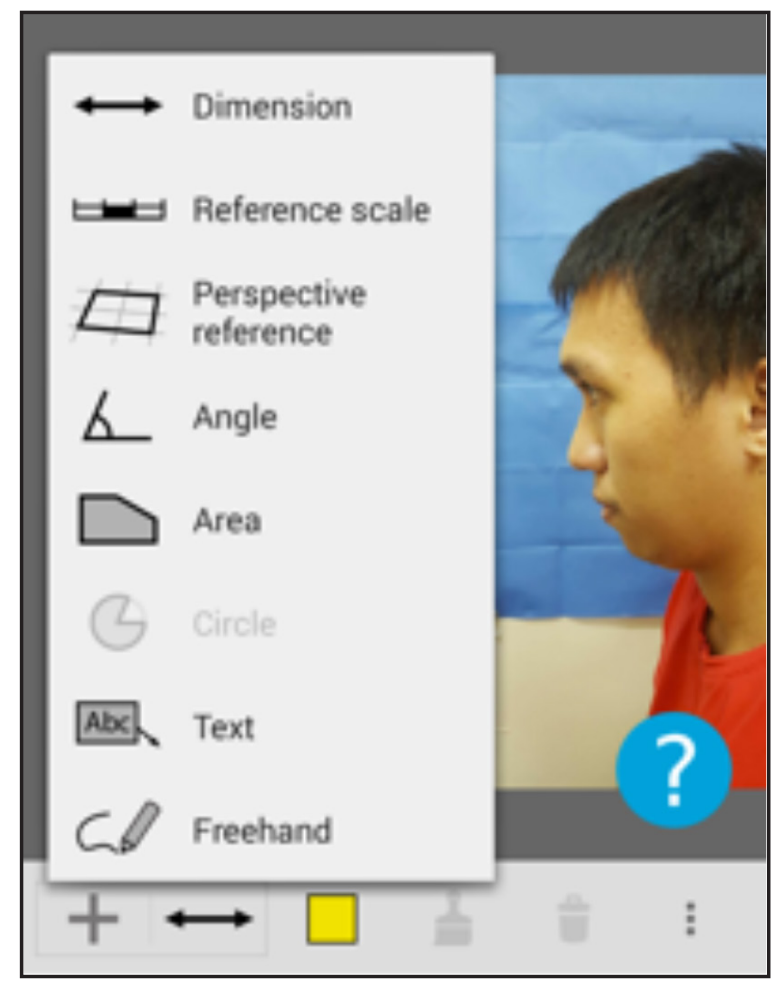

Figure 1. ImageMeter screen shot using a photo of one of our test patients, displaying the interactive options for analysis. (participant photo published in full with permission).
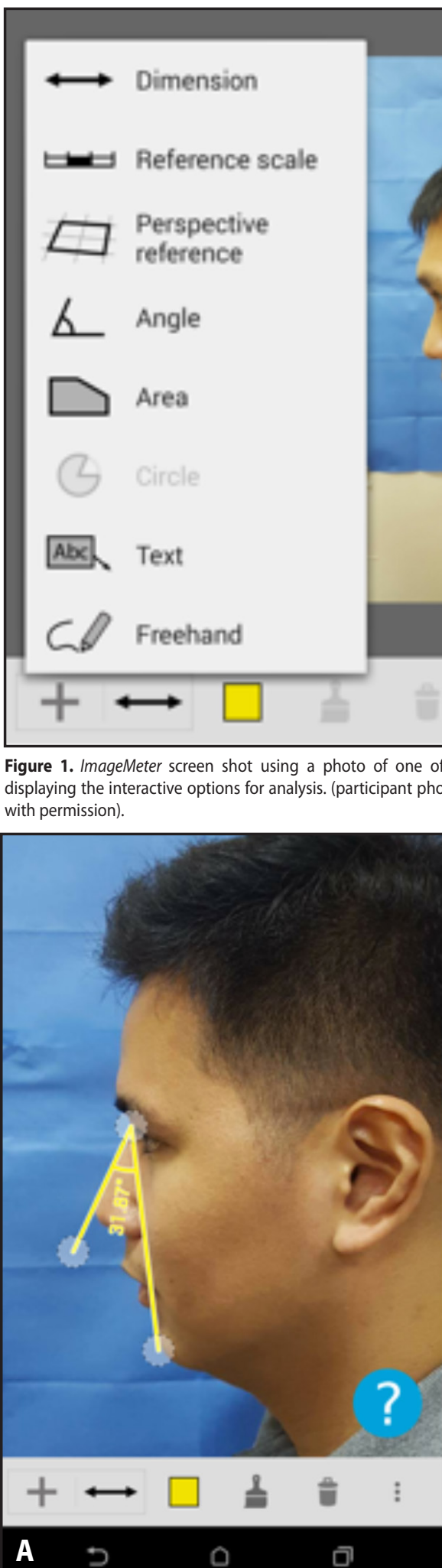

A

S

0
ㅁ
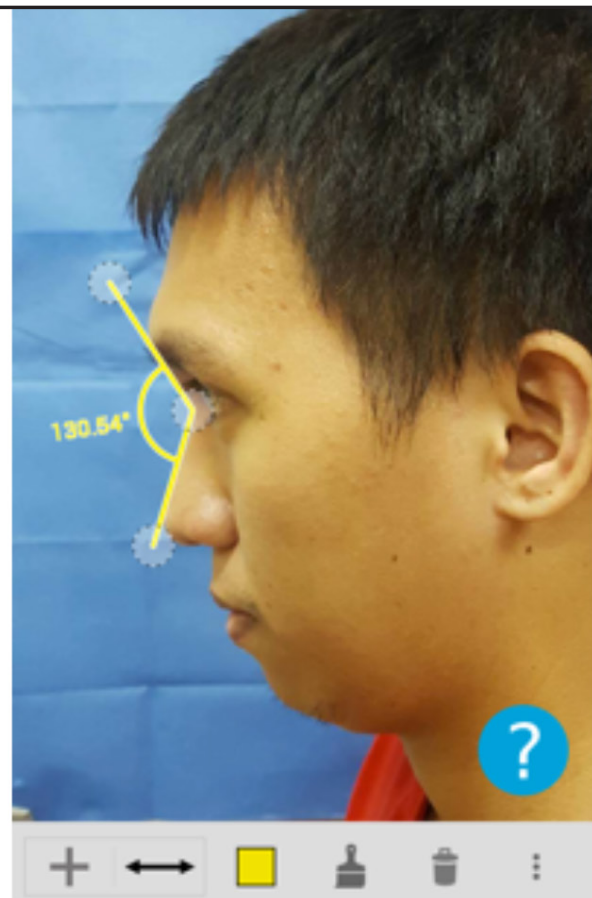

B

Figure 2. Facial angles measured A. Nasofacial angle B. Nasofrontal angle C. Nasolabial angle. Using the application's angle option, the respondent points and connects the facial landmarks per facia angle measured. The landmarks were adjusted as the respondent pleased and the angle generated automatically adjusted accordingly. (Photos of participants published in full with permission) 


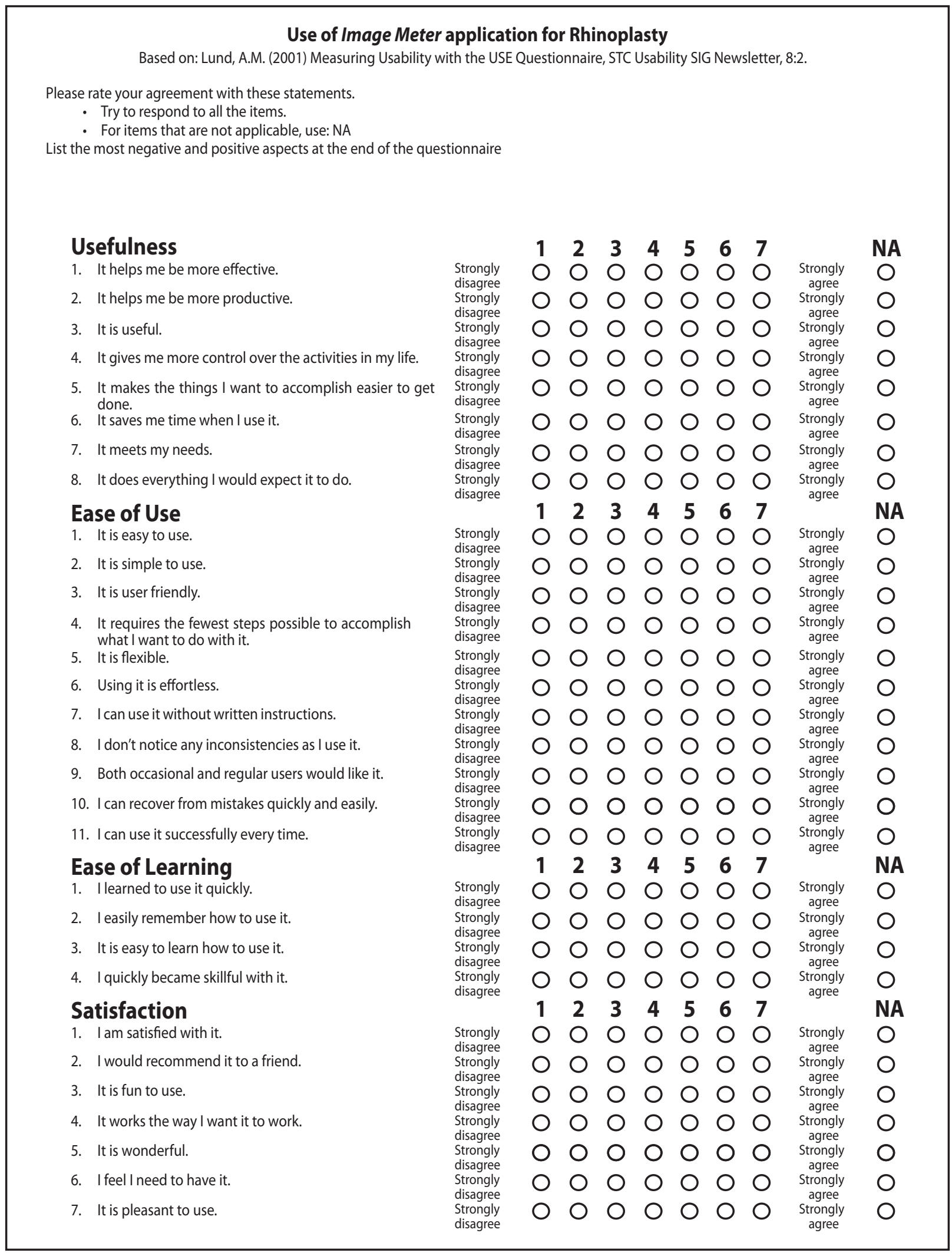

Figure 3. USE Questionnaire. (Reproduced with permission from Lund AM. Measuring Usability with the USE Questionnaire. STC Usability SIG Newsletter. 2001; 8(2): 3-6.) 


\section{ORIGINAL ARTICLES}

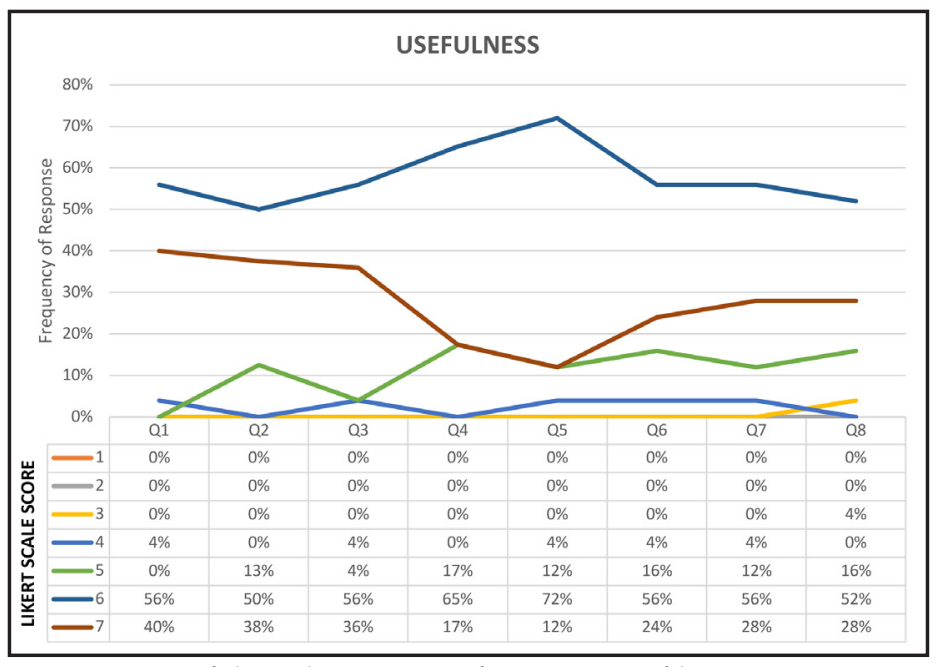

Figure 4. Frequency of Likert Scale score responses for questions on usefulness.

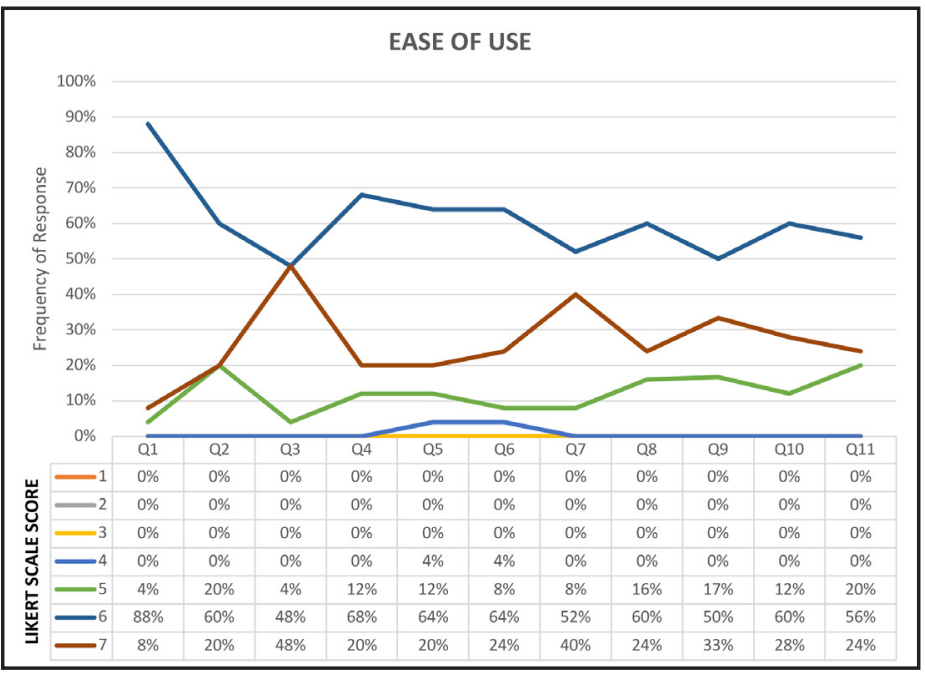

Figure 5. Frequency of Likert Scale score responses on ease of use.

\section{DISCUSSION}

The International Standards Organization (1994) defined usability as "the extent to which a product can be used by specified users to achieve specified goals with effectiveness, efficiency and satisfaction in a specified context of use". ${ }^{\prime 3}$ The findings of this study suggest that the ImageMeter has high usability with regards to its ease of use, satisfaction, usefulness and ease of learning.

Although software tools for facial analysis and measuring facial angles have been developed, ${ }^{4,14,15}$ our review of literature yielded no results on usability testing for these programs. In addition, we found no published studies on the use of the ImageMeter application for facial analysis. Moreover, the available software for measuring facial angles are desktop applications, making ImageMeter very attractive,

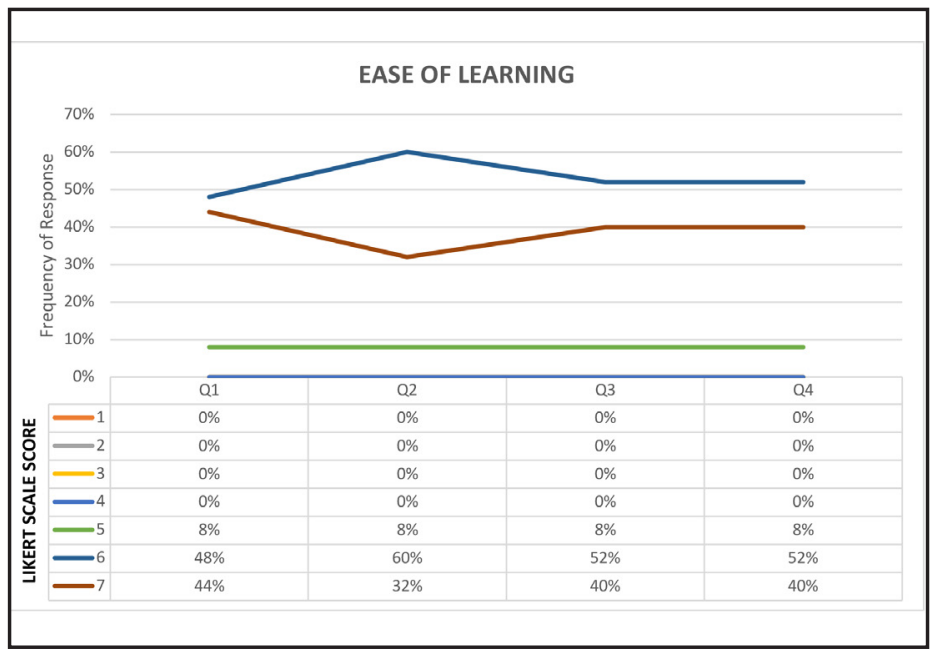

Figure 6. Frequency of Likert Scale score responses on ease of learning.

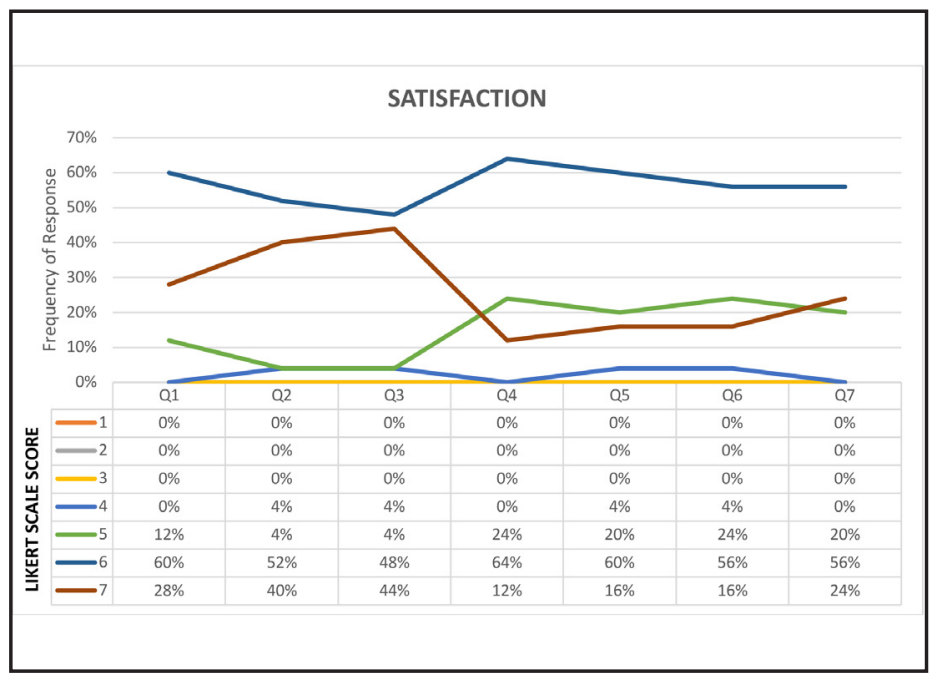

Figure 7. Frequency of Likert Scale score responses on satisfaction.

considering its convenience and portability, being a mobile phone application.

Although this study yielded a high usability rating by the respondents, the use of the app for facial analysis still poses a great number of limitations. First the analysis was subject to the inherent restrictions of the study design, including the lack of a control population, non-randomization and inability to control for confounding variables. Secondly there are no available studies on the accuracy and reproducibility of the measurements taken using the app, restricting the authors to make any comparisons. In addition, profile photograph analysis alone has many limitations including the type of camera and lens used, patient positioning, lighting, lens to subject distance, etc. Furthermore, while the answers of the participants 
showed internal consistency with no significant difference with regards to age and gender, it is still important to note that software literacy and familiarity with the use of mobile gadgets may contribute significantly to the success in the usage of the app. Finally, data on the respondents' ability to use the app including the correct identification of points and connection of points and accuracy of the angles generated were not measured, preventing the authors to make conclusions on the correctness of the respondents' use of the app.

It is therefore recommended that further studies on the accuracy and reproducibility of results on taking facial angle measurements using this app be done in a controlled and standardized manner. Moreover, the feature used from this application which is the measurement of angles is just one of its many possible uses in pre-operative evaluation. We recommend that the other features of this application be explored for possible use in other types of procedures/surgeries.

A usable product seeks to achieve three main outcomes: (1) the product is easy for users to become familiar with and competent in using it during the first contact, (2) the product is easy for users to achieve their objective through using it, and (3) the product is easy for users to recall the user interface and how to use it on later visits. ${ }^{16}$ Our results showed that for the respondents, the app was easy to use, easy to learn, and satisfactory, therefore usable.

In conclusion, our results suggest that when applied to human facial analysis, the ImageMeter may be a usable tool for ENT surgeons in the pre-operative evaluation of patients undergoing rhinoplasty.

\section{ACKNOWLEDGEMENTS}

The authors would like to acknowledge the participation and cooperation of the St. Luke's Medical Center, Department of ENT-HNS residents and consultants for their patience in learning the app and answering the questionnaire, as well as the Out-Patient Clinic staff for allowing the authors to perform the study at the clinic.

\section{REFERENCES}

1. Sheckter CC, Kane JT, Minneti M, Garner W, Sullivan M, Talving P, et al. Incorporation of fresh tissue surgical simulation into plastic surgery education: maximizing extraclinical surgical experience. J Surg Educ. 2013 Jul-Aug; 70(4): 466-74. DOI: 10.1016/j.jsurg.2013.02.008; PMID: 23725934.

2. Chivers QJ, Ahmad J, Lista F, Warren RJ, Arkoubi AY, Mahabir RC, et al. Cosmetic surgery training in Canadian plastic surgery residencies: are we training competent surgeons? Aesthet Surg J. 2013 Jan; 33(1): 160-165. DOI: 10.1177/1090820X12467794; PMID: 23169820

3. Oni G, Ahmad J, Zins JE, Kenkel JM. Cosmetic surgery training plastic surgery residency programs in the united states: how have we progressed in the last three years? Aesthet Surg J. 2011 May; 31(4), 445-455. DOI: 10.1177/1090820X11404551; PMID: 21551438

4. Ozkul T, Ozkul H, Akhtar R, Al-Kaabi F, Jumaia T. A Software Tool for Measurement of Facial Parameters. The Open Chemical and Biomedical Methods Journal. 2009; 2(1): 69-74. DOI: 10.2174/1875038900902010069.

5. DiBernardo BE, Adams RL, Krause J, Fiorillo MA, Gherardini G. Photographic standards in plastic surgery. Plast Reconstr Surg. 1998 Aug; 102(2): 559-568. PMID: 9703100.

6. Ventola CL. Mobile devices and apps for health care professionals: uses and benefits. PT. 2014 May; 39(5): 356-364. PMID: 24883008 PMCID: PMC4029126.

7. Aungst TD. Medical applications for pharmacists using mobile devices. Ann Pharmacother. 2013 Jul-Aug. 47(7-8). DOI: 10.1345/aph.1S035; PMID: 23821609

8. Divali P, Camosso-Stefinovic J, Baker R. Use of personal digital assistants in clinical decision making by health care professionals: a systematic review. Health Informatics J. $2013 \mathrm{Mar} ; 19(1)$, 16-28. DOl: 10.1177/1460458212446761; PMID: 23486823.

9. Murfin M. Know your apps: an evidence-based approach to evaluation of mobile clinical applications. J Physician Assist Educ. 2013; 24(3), 38-40. PMID: 24261171.

10. Mickan S, Tilson JK, Atherton $\mathrm{H}$, Roberts NW, Heneghan C. Evidence of effectiveness of health care professionals using handheld computers: a scoping review of systematic Research. Med Internet Res. 2013 Oct; 15(10), e212. DOI: 10.2196/jmir.2530; PMID: 24165786 PMCID: PMC3841346.

11. Farin D. ImageMeter - photo measure - apps on Google Play [Internet]. 2017[cited 2019 Feb 3]. Retrieved from Google Play: https://play.google.com/store/apps/details?id=de.dirkfarin imagemeter\&hl=en

12. Lund AM. Measuring usability with the USE questionnaire. STC Usability SIG Newsletter; 2001; 8(2): 3-6.

13. International Standards Organization. Ergonomic requirements for office work with visual display terminals. Part 11: Guidance on usability (ISO DIS 9241-11). London: International Standards Organization;1994.

14. Loveday OE., Hakeem F B, Lekara D T. A Software Tool for Facial Analysis. Research Journal of Applied Sciences, Engineering and Technology.2012;4(6): 551-556.

15. Tollefson TT, Sykes JM. Computer Imaging Software for Profile Photograph Analysis. Archives of Facial Plastic Surgery 2007 Mar-Apr; 9(2): 113-119. DOI: 10.1001/archfaci.9.2.113; PMID: 17372065 .

16. Interaction-Design.org, What is Usability? n.d. [cited 2017 Oct]. Available from: https://www. interaction-design.org/literature/topics/usability. 